\title{
Spindle and kinetochore associated complex subunit 1 regulates the proliferation of oral adenosquamous carcinoma CAL-27 cells in vitro
}

\author{
Bin Zhang ${ }^{1,2}$, Ke Yi Li ${ }^{2}$, Hai Ying Chen ${ }^{3}$, Shao Dong Pan ${ }^{3}$, Li Cheng Jiang ${ }^{2}$, Ya Ping Wu ${ }^{3,4^{*}}$ and Shu Wei Liu ${ }^{1^{*}}$
}

\begin{abstract}
Background: The prognosis of oral squamous cell carcinoma is very poor due to local recurrence and metastasis. This study explores the molecular events involved in oral carcinoma with the goal of developing novel therapeutic strategies. The mitotic spindle is a complex mechanical apparatus required for the accurate segregation of sister chromosomes during mitosis. Spindle and kinetochore associated complex subunit 1 (SKA1) is a microtubulebinding subcomplex of the outer kinetochore that is essential for proper chromosome segregation. In recent years, much attention has been focused on determining how SKA proteins interact with each other, as well as their biological role in cancer cells. However, the precise role of SKA1 in oral carcinoma remains unknown.
\end{abstract}

Methods: In order to investigate the role of SKA1 in oral cancer, we employed lentivirus-mediated shRNA to silence SKA1 expression in the CAL-27 human oral adenosquamous carcinoma cell line.

Results: Depletion of SKA1 in CAL-27 cells significantly decreased cell proliferation, as determined by MTT and colony formation assays. These results strongly demonstrate that reduced SKA1 protein levels may cause inhibition of tumor formation. The shRNA-mediated depletion of SKA1 also led to G2/M phase cell cycle arrest and apoptosis.

Conclusion: This is the first report to show that SKA1 plays an important role in the progression of oral adenosqamous carcinoma. Thus, silencing of SKA1 by RNAi might be a potential therapy for this disease.

Keywords: Oral adenosquamous carcinoma, CAL-27 cells, Cell proliferation, SKA1, RNAi

\section{Background}

Worldwide, there are an estimated 405,000 new cases of oral cancer diagnosed each year [1]. Despite advances in the therapeutic management of squamous cell carcinoma over the last few decades, the prognosis is still very poor due to local recurrence and metastasis [2-4]. This disappointing outcome, even with existing therapies, strongly suggests that novel targeted therapeutic agents are needed to improve the treatment of patients diagnosed with oral cancer. The successful use of small interfering RNA (RNAi) to downregulate gene expression in several model systems has led to many attempts to explore this

\footnotetext{
*Correspondence: ywu@umcutrecht.nl; Ishw@sdu.edu.cn

${ }^{3}$ Key Laboratory of Oral and Maxillofacial-Head and Neck Medicine, Central Laboratory, Liaocheng People's Hospital and Liaocheng Clinical School, Liaocheng, Shandong 252000, P. R. China

'Department of Anatomy, Shandong University, School of Medicine, Jinan, Shandong 250012, P. R. China

Full list of author information is available at the end of the article
}

methodology in cancer therapeutic settings [5]. RNAi is a post-transcriptional mechanism of gene silencing through chromatin remodeling, inhibition of protein translation, or direct mRNA degradation, which is ubiquitous in eukaryotic cells [6]. Along these lines, several recent studies have documented the successful downregulation of gene expression, resulting in increased apoptosis and decreased proliferation in many cancer cell lines [4,7]. Long-lasting RNAi-based gene silencing can be achieved using lentivirus-based expression systems, which drive the production of short hairpin RNAs (shRNAs) [8]. Lentiviral vectors have become a promising tool for the establishment of transgenic animals and manipulation of the mammalian genome [9].

Mitotic chromosome segregation requires kinetochores to generate physical connections between chromosomes and spindle microtubule polymers [10]. Notably, spindle

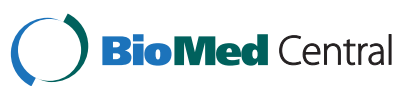


and kinetochore-associated protein complexes have several key properties, and play an important role in coupling chromosome movement to microtubule depolymerization $[11,12]$. Spindle and kinetochore associated complex subunit 1 (SKA1) is a microtubule-binding subcomplex of the outer kinetochore that is essential for proper chromosome segregation $[13,14]$. Depletion of SKA1 proteins results in sparse microtubule arrays that often exhibited twisted or bent spindles. The chromosomes appear to interact with the spindle microtubule, but fail to organize into metaphase plates $[15,16]$. Much attention has been focused in recent years on how SKA proteins interact with each other, and their biological role in cancer cells. However, until now, the precise role of SKA1 in oral carcinoma has remained unclear. Therefore, the goal of this study was to determine if SKA1 plays a significant role in oral adenosquamous cell carcinoma by using lentivirus-mediated shRNA for the functional gene knockdown of SKA1 expression in CAL-27 cells.

\section{Results and discussion Method validation}

We used recombinant lentivirus harboring shRNA for SKA1 to infect CAL-27 cells. The successful infection and efficiency were confirmed by evaluating GFP expression levels. After infection, approximately $90 \%$ of cells infected with Lv-shSKA1 expressed GFP (Figure 1).

These results suggest that the lentivirus infections are successful and highly efficient. The effect of Lv-shSKA1 on SKA1 expression in CAL-27 cells was observed by western blot analysis (Figure 2).

Western blot analysis showed that SKA1 protein was not expressed in Lv-shSKA1 -infected cells. In contrast, equal protein expression was observed in uninfected cells and Lv-shCon-infected (negative control) cells.

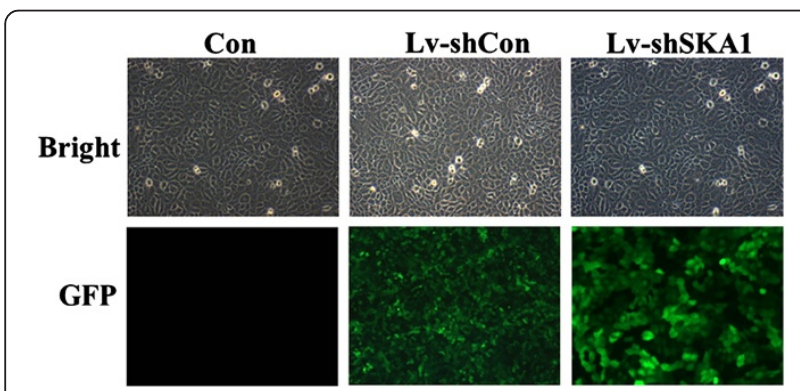

Figure 1 Infection of cells with lentiviral shRNA targeting SKA1 in CAL-27 cells. GFP expression in uninfected (Con), LV-shSKA1infected, and LV-shCon-infected CAL-27 cells indicates lentivirus infection efficiency in CAL-27 cells. Transduction efficiency was estimated 6 days after infection at $\mathrm{MOI}$ of 10. Light micrograph (left); Fluorescent micrograph (right) (200x).

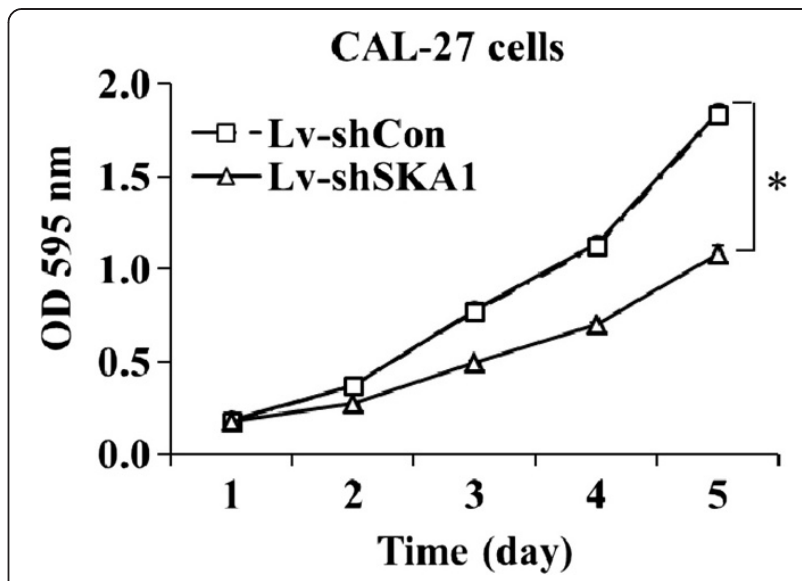

Figure 2 Western blot analysis of SKA1 protein expression levels in Lv-shSKA1-infected, LV-shCon-infected and uninfected CAL-27 cells (Con). ${ }^{*} p<0.01$. Data are representative of three independent experiments.

Therefore, Lv-shSKA1 specifically and strongly abolished SKA1 expression in CAL-27 cells.

\section{SKA1 affects CAL-27 cell proliferation}

To elucidate the functional role of SKA1 in oral adenosquamous carcinoma cells, we examined the effect of SKA1 knockdown on the proliferation and colony formation of CAL-27 cells. Cell proliferation was evaluated by the MTT assay, and the results showed that downregulation of SKA1 expression in CAL-27 cells significantly decreased cell viability. Five days postinfection, the viability of Lv-shSKA1-infected CAL-27 cells decreased compared to Lv-shCon-infected cells $(p<0.01$; Figure 3$)$. Cell viability was the same in LvshCon-infected and uninfected CAL-27 cells.

\section{Downregulation of SKA1 in CAL-27 cells significantly reduces colony formation}

To determine the role of SKA1 in human oral adenosquamous tumorigenesis in vitro, we analyzed CAL-27 tumor cell colony formation in a soft agar assay. Downregulation of SKA1 in CAL-27 cells caused a substantial reduction in colony formation (Figures 4 and 5) compared with uninfected and Lv-shCon-infected cells $(p<0.01)$.

Results from the colony formation assay strongly indicate that reduced SKA1 protein levels might result in significant inhibition of tumor formation in human oral adenosquamous cancer cells. Furthermore, the data showed that SKA1 was critical for the proliferation and tumorigenicity of human oral adenosquamous cancer cells in vitro. 

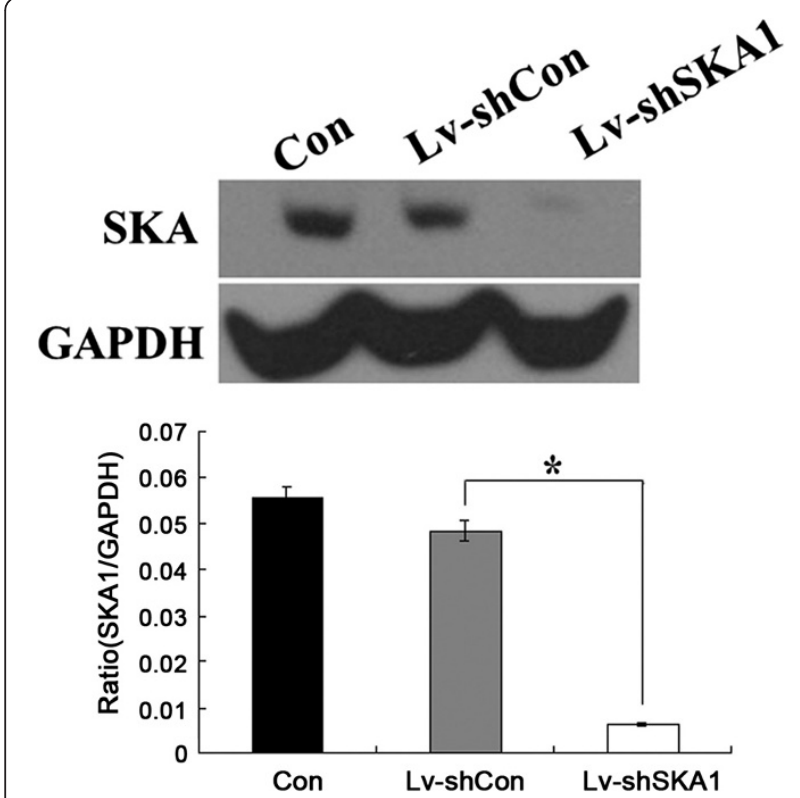

Figure 3 SKA1 affects the proliferation of CAL-27 cells. CAL-27 cells were seeded into 96-well plates at a density of $2.5 \times 10^{3}$ cells/ well in triplicate, and proliferation rates were measured by the MTT assay. Data are representative of three independent experiments. ${ }^{*} p<0.01$

\section{SKA1 depletion affects the cell cycle of CAL-27 cells}

Flow cytometry was used to determine the effect of LvshSKA1 infection on the cell cycle of CAL-27 cells. As shown in Table 1, down-regulation of SKA1 led to an arrest in G 1/G 0 and $\mathrm{G} 2 / \mathrm{M}$ phase, the percentage of cells in $S$ phase reduced and sub-G 1 appeared. This cell cycle analysis result suggesting the sequential events of cell cycle arrest by SKA1 silencing in CAL-27 cells is followed by apoptosis process. However, there were no significant differences in cell cycle distribution between in noninfected (Con) group and $\mathrm{Lv}$-shCon group.

\section{Discussion}

Upon entry into mitosis, the microtubule network is rearranged to form the mitotic spindle, which results in segregation of sister chromatids [17-19]. Central to this process is the proper attachment of spindle microtubules to kinetochores, proteinaceous structures assembled on centromeric chromatin [20]. Three subunits of the SKA1 complex (SKA1, SKA2, and SKA3/RAMA) are direct components of the kinetochore-microtubule interface [18]. SKA2 protein requires SKA1, a protein-binding partner, for stability, and SKA2 is required for the correct assembly of SKA1 on the kinetochore [20]. It is well established that depletion of the SKA complex leads to apoptosis in many mammalian cell lines. Here, we evaluated the effect of SKA1 depletion in CAL-27 cells. Our

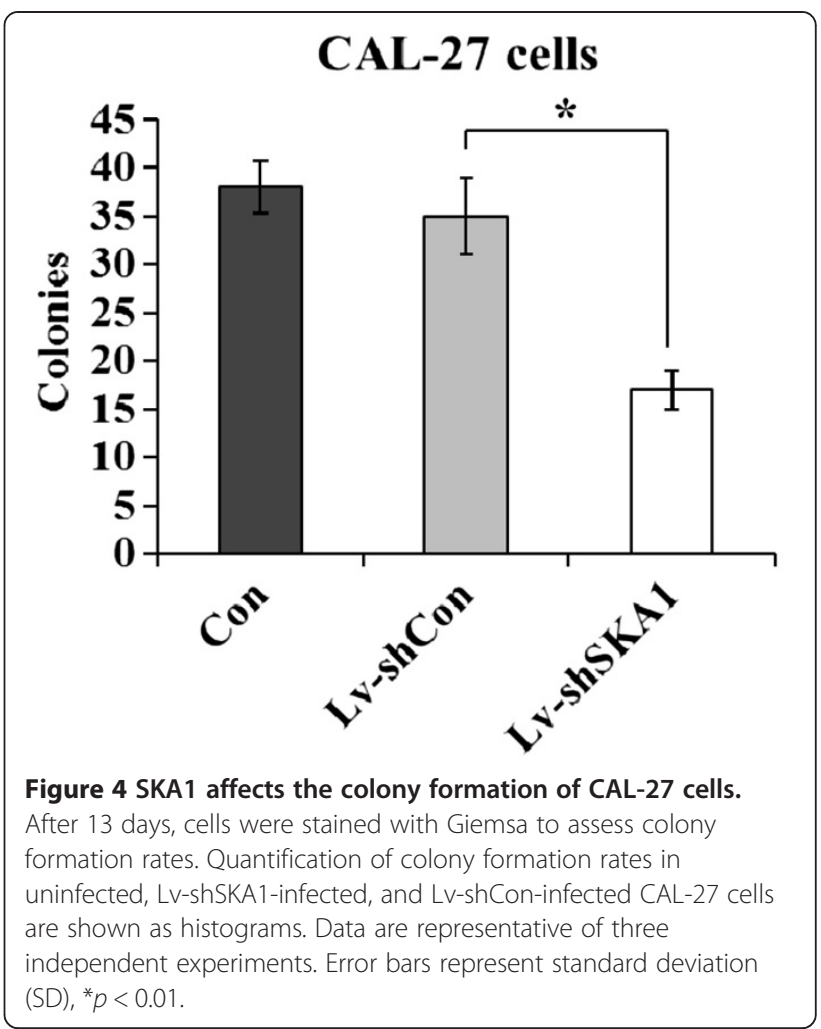

results showed that knockdown of SKA1 expression led to reduced cell viability and increased apoptosis, as anticipated.

To date, there are very few reports on the role of SKA proteins in cancer formation and metastasis [21,22]. Clonogenic side population cells in some cancers, including multiple human myeloma and primary tumors, exhibit tumor-initiating characteristics. Gene expression analysis showed that clonogenic side population cells in cancers express genes involved in the cell cycle and mitosis (e.g., SKA1, CCNB1, CDC25C, CDC2, BIRC5, CENPE, AURKB, KIFs, TOP2A, ASPM) more strongly than non-side population cells [23]. In accordance with these reports, we found that SKA1 appears to be involved in oral carcinoma by regulating spindle checkpoint silencing and maintaining chromosome cohesion in mitosis. To the best of our knowledge, this study is the first to report that SKA1 expression is important in oral adenosquamous cancer cell proliferation. These findings will significantly enhance the current understanding of the role of the SKA complex in cancer cell proliferation. It is worth noting that the present data are only applicable to oral adenosquamous carcinomas. Thus, studies in other cell lines could be of interest. Moreover, future studies are needed to determine the precise mechanism underlying SKA1 regulation of oral adenosquamous cancer cell proliferation. 


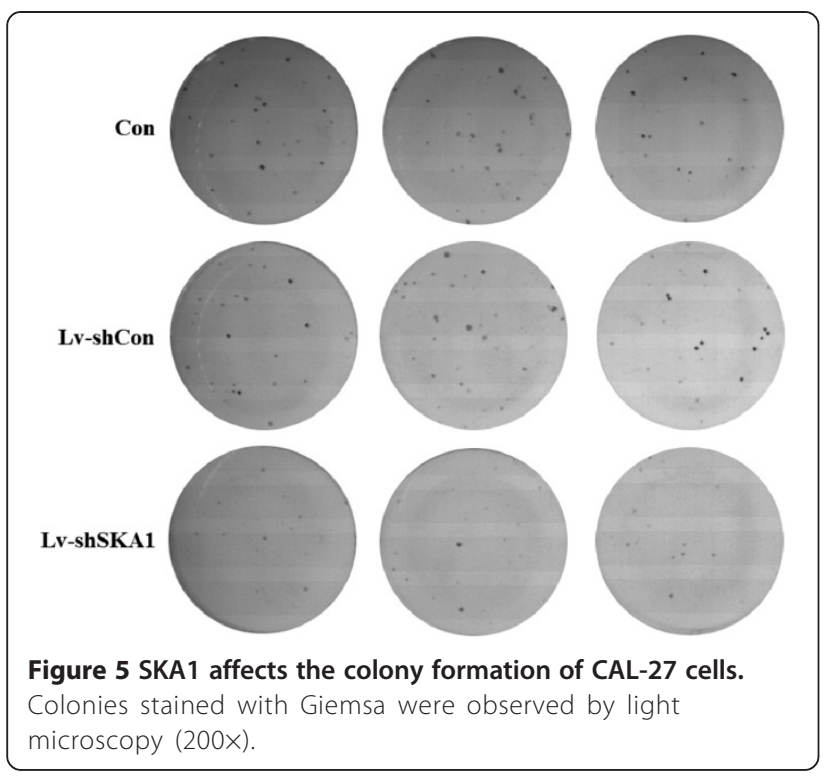

\section{Conclusions}

This is the first report to show that SKA1 is important in the proliferation of oral adenosquamous cells. Thus, SKA1 silencing by RNAi might be a potential therapy for oral carcinoma.

\section{Methods}

\section{Cell culture}

CAL-27 and HEK 293 T cells were maintained in Dulbecco's Modified Eagle's Medium (DMEM) containing 10\% Fetal Bovine Serum (FBS), and $4 \mathrm{mM}$ L-glutamine, adjusted to contain $3.7 \mathrm{~g} / \mathrm{l}$ sodium bicarbonate and $4.5 \mathrm{~g} / \mathrm{l}$ glucose (Hyclone, Logan, UT). Cell cultures were maintained as a monolayer in $75 \mathrm{~cm}^{2}$ tissue-culture treated flasks (Bedford, MA) at $37^{\circ} \mathrm{C}$ in a humidified atmosphere of $5 \% \mathrm{CO}_{2}$.

\section{Lentivirus production and infection of CAL-27 cells}

The cDNA sequence of SKA1 was obtained from GenBank (accession number: NM_001039535). ShRNA for SKA1 (5'-CCG-GCC-TGA-CAC-AAA-GCT-CCT-

Table 1 SKA1 depletion affects the cell cycle of CAL-27 cells

\begin{tabular}{ccccc}
\hline Phase & G0/G1 & S & G2/M & Sub-G1 \\
\hline Con & $59.03 \pm 1.59$ & $29.53 \pm 2.66$ & $11.40 \pm 1.04$ & $0.45 \pm 0.06$ \\
Lv-shCon & $56.33 \pm 0.38$ & $31.60 \pm 0.78$ & $12.07 \pm 0.75$ & $0.43 \pm 0.06$ \\
Lv-shSKA1 & $61.73 \pm 1.53^{* *}$ & $19.27 \pm 1.52$ & $19.03 \pm 0.49^{*}$ & $1.00 \pm 0.06^{*}$ \\
\hline
\end{tabular}

The flow cytometric analysis of cell cycle of uninfected CAL-27 cells (Con), Lv-shSKA1, and Lv-shCon. The percentages of the cells in each phase of the cycle are presented as the mean \pm SD of three independent experiments. *represents $p<0.05$ between Lv-shSKA1group and Lv-shCon group.

**represents $p<0.01$ between Lv-shSKA1group and Lv-shCon group. There were no significant differences in cell cycle distribution between noninfected (Con) group and Lv-shCon group ( $p>0.05$ ).
AAA-TCT-CGA-GAT-TTA-GGA-GCT-TTG-TGT-CAGGTT-3') was inserted into the lentivirus expression plasmid pFH-L (Shanghai Hollybio, China). Non-silencing shRNA (5' ${ }^{\prime}$ TTC-TCC-GAA-CGT-GTC-ACG-T-3') was used as a control. In order to generate SKA1-shRNA and control lentivirus, lentiviral shRNA plasmids targeting SKA1 or a control were transfected into HEK293T cells using Lipofectamine 2000 (Invitrogen, Carlsbad, CA) together with the gag/pol packaging vector (pCMV $\Delta$ R8.92, Shanghai Hollybio, China) and the pVSVG-1 encoding plasmid (Shanghai Hollybio) according to the manufacturer's instructions. For lentivirus infection, CAL-27 cells were grown at a density of $5 \times 10^{4}$ cells/well and infected with SKA1-shRNA lentivirus or control lentivirus at a MOI of 10.

\section{Determine the infection efficiency of SKA1-shRNA lentivirus}

To determine the infection efficiency of SKA1-shRNA lentivirus, CAL-27 cells expressing GFP protein were observed by fluorescence microscopy (CKX41, Olympus, Tokyo-Japan).

For western blot analysis, CAL-27 cells were grown for 6 days. The cells were washed twice with ice-cold PBS, scraped, centrifuged, and lysed in RIPA buffer with PMSF (Beyotime Biotechnology, Jiangsu, China). Cell extracts were prepared on ice by ultrasonic disruption. After centrifugation at $12,000 \times g$ for $15 \mathrm{~min}$ at $4^{\circ} \mathrm{C}$, the supernatants were collected, and the protein concentrations were determined using the BCA Protein Assay Kit. Cell lysates were dissolved in 2x SDS sample buffer (100 mM Tris-HCl pH 6.8, 10 mM EDTA, 4\% SDS, 10\% glycine). Equal amounts of protein were resolved on $10 \%$ SDS-PAGE gels and electrophoretically transferred onto polyvinylidene fluoride membranes. Membranes were blocked in 5\% skim milk and hybridized with primary antibodies (1:1000 dilution; SBA3500101, Sigma-Aldrich, St. Louis, MO, USA). After incubation with horseradishperoxidase-conjugated secondary antibody (1:5000 dilution; sc-2004, Santa Cruz Biotechnology, Santa Cruz, CA, USA) at room temperature, immunoreactive proteins were detected using a Chemiluminescent ECL Assay Kit (Amersham Pharmacia Biotech, UK), according to the manufacturer's instructions. GAPDH protein levels were used as a control to verify equal protein loading. Band intensities were analyzed with Quantity One analysis software (Bio-Rad, Hercules, CA, USA).

\section{MTT cell viability assay}

In brief, both uninfected and infected CAL-27 cells $\left(2.5 \times 10^{3}\right.$ cells/well) were seeded in 96-well plates. After $1,2,3,4$, or 5 days of infection, $100 \mu \mathrm{l}$ MTT $(5 \mathrm{mg} / \mathrm{ml})$ was added, followed by incubation for another $4 \mathrm{~h}$ at $37^{\circ} \mathrm{C}$. The reaction was stopped by replacing the MTT- 
containing medium with $100 \mu \mathrm{l}$ acidic isopropanol (10\% SDS, $5 \%$ isopropanol, $0.01 \mathrm{~mol} / \mathrm{l} \mathrm{HCl}$ ), and the formazan salts were dissolved by gentle shaking for about $10 \mathrm{~min}$ at room temperature. For colorimetric analysis, the absorbance at $595 \mathrm{~nm}$ was recorded using a microplate reader (Bio-Rad). The optical of sample bands were analyzed with Quantity One analysis software (Bio-Rad, USA). Each assay was repeated at least three times. Relative cell viability was compared to the untreated (blank) group.

\section{Colony formation assay}

Agar medium (0.8\%) was prepared in 6-well plates. Uninfected and infected CAL-27 cells were trypsinized, centrifuged, resuspended in $0.4 \%$ agar medium (equal volumes of $0.8 \%$ agar and culture medium), and plated onto the top agar at 200 cells/well. Cell culture medium was changed regularly. After 13 days of culture, adherent cells were washed twice with PBS, and fixed in 4\% paraformaldehyde for $30 \mathrm{~min}$ at room temperature. Colonies were stained with Giemsa solution for $72 \mathrm{~h}$, washed with water, and air-dried. The colonies were counted under a fluorescence microscope.

\section{Cell cycle analysis}

Uninfected and infected CAL-27 cells were seeded in 6-well culture plates at a density of $5 \times 10^{4}$ cells/well, and incubated in complete medium to $70 \%$ confluence. The cells were harvested, washed twice with ice-cold PBS, and fixed in $75 \%$ cold ethanol at $4{ }^{\circ} \mathrm{C}$ overnight. Propidium iodide (PI) staining of nuclei was used to monitor the cell cycle phases. The fluorescence of DNA-bound PI in cells was measured with a Cell Lab Quanta Beckman Coulter (Beckman Coulter, Miami, FL, USA), and data were analyzed using Multi-Cycle AV software (Phoenix Flow Systems, San Diego, CA, USA).

\section{Statistical analysis}

PASW Statistics 18, Prism version 4.00 for Windows, and InStat 3.0 software were used for statistical data analyses. Numerical data were analyzed by one-way ANOVA. Data are represented as mean and standard error. Turkey's test was used for multiple comparisons. $\mathrm{P}$ values less than 0.05 were considered statistically significant.

\footnotetext{
Abbreviations

SKA1: Spindle and kinetochore associated complex subunit 1; RT-PCR: Realtime reverse transcription-polymerase chain reaction; siRNA: Small interfering double stranded RNA; shRNA: Short hairpin; LV-shNLK: Lentivirus/shRNA-NLK; LV-shCon: Letivirus/shRNA-Control.
}

\section{Competing interests}

The authors declare that they have no competing interests.

\section{Authors' contributions}

$B Z$, and SWL have designed the project. In vitro determinations have been performed by BZ and KL Acquisitions of data: laboratory or clinical/literature search have been performed by HYC and SDP. Statistical study has been performed by LCJ. Drafting of the article and/or critical revision have been performed by BZ and YPW. Final approval and guarantor of manuscript have been performed by SWL. All authors read and approved the final manuscript.

\section{Acknowledgements}

The National Natural Science Foundation of China funded project is acknowledged for the financial support for this work (81272958).

\section{Author details}

${ }^{1}$ Department of Anatomy, Shandong University, School of Medicine, Jinan, Shandong 250012, P. R. China. ${ }^{2}$ Department of Oral and Maxillofacial Surgery, Liaocheng People's Hospital, Liaocheng, Shandong 252000, P. R. China. ${ }^{3}$ Key Laboratory of Oral and Maxillofacial-Head and Neck Medicine, Central Laboratory, Liaocheng People's Hospital and Liaocheng Clinical School, Liaocheng, Shandong 252000, P. R. China. ${ }^{4}$ Department of Clinical Chemistry and Haematology, University Medical Centre Utrecht, G03.550, Heidelberglaan 100, 3584 CX, Utrecht, The Netherlands.

Received: 4 July 2013 Accepted: 19 August 2013

Published: 20 August 2013

\section{References}

1. Ganly I, Patel S, Shah J: Early stage squamous cell cancer of the oral tongue-clinicopathologic features affecting outcome. Cancer 2012, 118:101-111.

2. Warnakulasuriya S: Global epidemiology of oral and oropharyngeal cancer. Oral Oncol 2009, 45:309-316.

3. Leung KW, Yang KC, Chen CJ: Adenosquamous Carcinoma of Buccal Mucosa. Taiwan J Oral Maxillofac Surg 2009, 20:19-28.

4. Zhang D, Chen ZG, Liu SH, Dong ZQ, Dalin M, Bao SS, et al: Galectin-3 gene silencing inhibits migration and invasion of human tongue cancer cells in vitro via downregulating $\beta$-catenin. Acta Pharmacol Sin 2013, 34:176-184.

5. Lima RT, Martins LM, Guimarães JE, Sambade C, Vasconcelos MH: Specific downregulation of bcl-2 and XIAP by RNAi enhances the effects of chemotherapeutic agents in MCF-7 human breast cancer cells. Cancer Gene Ther 2004, 11:309-316.

6. Pai $S$, Lin $Y$, Macaes B, Meneshian A, Hung C, Wu T: Prospects of RNA interference therapy for cancer. Gene Ther 2005, 13:464-477.

7. Akar U, Chaves-Reyez A, Barria M, et al: Silencing of Bcl-2 expression by small interfering RNA induces autophagic cell death in MCF-7 breast cancer cells. Autophagy 2008, 4:669-679.

8. Park F: Lentiviral vectors: are they the future of animal transgenesis? Physiol Genomics 2007, 31:159-173.

9. Kumar LD, Clarke AR: Gene manipulation through the use of small interfering RNA (siRNA): From in vitro applications. Adv Drug Deliv Rev 2007, 59:87-100

10. Cheeseman IM, Desai A: Molecular architecture of the kinetochoremicrotubule interface. Nat Rev Mol Cell Biol 2008, 9:33-46.

11. Tanaka TU, Desai A: Kinetochore-microtubule interactions: the means to the end. Curr Opin Cell Biol 2008, 20:53-63.

12. Welburn JPI, Grishchuk EL, Backer CB, Wilson-Kubalek EM, Yates lii JR, Cheeseman IM: The human Kinetochore Ska1 complex facilitates microtubule depolymerization-coupled motility. Dev Cell 2009, 16:374-385

13. Hanisch A, Silljé HH, Nigg EA: Timely anaphase onset requires a novel spindle and kinetochore complex comprising Ska1 and Ska2. EMBO J 2006, 25:5504-5515.

14. Schmidt JC, Arthanari H, Boeszoermenyi A, Dashkevich NM, Wilson-Kubalek EM, Monnier N, et al: The Kinetochore-Bound Ska1 complex tracks depolymerizing microtubules and binds to curved protofilaments. Dev Cell 2012, 23:968-980.

15. Rines DR, Gomez-Ferreria MA, Zhou Y, DeJesus $P$, Grob S, Batalov $S$, et al: Whole genome functional analysis identifies novel components required for mitotic spindle integrity in human cells. Genome Biol 2008, 9:R44. 1-12 
16. Gaitanos TN, Santamaria A, Jeyaprakash AA, Wang B, Conti E, Nigg EA: Stable kinetochore-microtubule interactions depend on the Ska complex and its new component Ska3/C130rf3. EMBO J 2009, 28:1442-1452

17. Gladfelter A, Berman J: Dancing genomes: fungal nuclear positioning. Nat Rev Microbiol 2009, 7:875-886.

18. Kops GJ, Saurin AT, Meraldi P: Finding the middle ground: how kinetochores power chromosome congression. Cell Mol Life Sci 2010, 67:2145-2161.

19. Guimaraes GJ, DeLuca JG: Connecting with Ska, a key complex at the kinetochore-microtubule interface. EMBO J 2009, 28:1375-1377.

20. Maiato H, DeLuca J, Salmon E, Earnshaw WC: The dynamic kinetochoremicrotubule interface. J Cell Sci 2004, 117:5461-5477.

21. Rice L, Waters CE, Eccles J, Garside H, Sommer P, Kay P, et al: Identification and functional analysis of SKA2 interaction with the glucocorticoid receptor. J Endocrinol 2008, 198:499-509.

22. Daum JR, Wren JD, Daniel JJ, Sivakumar S, McAvoy JN, Potapova TA, et al: Ska3 is required for spindle checkpoint silencing and the maintenance of chromosome cohesion in mitosis. Curr Biol 2009, 19:1467-1472.

23. Nara M, Teshima K, Watanabe A, Ito M, Iwamoto K, Kitabayashi A, et al: Bortezomib reduces the tumorigenicity of multiple myeloma via downregulation of upregulated targets in clonogenic side population cells. PLoS One 2013, 8:e56954.

doi:10.1186/1475-2867-13-83

Cite this article as: Zhang et al: Spindle and kinetochore associated complex subunit 1 regulates the proliferation of oral adenosquamous carcinoma CAL-27 cells in vitro. Cancer Cell International 2013 13:83.

\section{Submit your next manuscript to BioMed Central and take full advantage of:}

- Convenient online submission

- Thorough peer review

- No space constraints or color figure charges

- Immediate publication on acceptance

- Inclusion in PubMed, CAS, Scopus and Google Scholar

- Research which is freely available for redistribution 